\title{
BMJ open Risk of cancer in patients using glucose- lowering agents: a nationwide cohort study of 3.6 million people
}

\author{
Charlotte Andersson, ${ }^{1}$ Allan Vaag, ${ }^{2}$ Christian Selmer, ${ }^{1}$ Michelle Schmiegelow, ${ }^{1}$ \\ Rikke Sørensen, ${ }^{1}$ Jesper Lindhardsen, ${ }^{1}$ Gunnar H Gislason, ${ }^{1}$ Lars Køber, ${ }^{3}$ \\ Christian Torp-Pedersen ${ }^{1,4}$
}

To cite: Andersson C, Vaag A, Selmer C, et al. Risk of cancer in patients using glucose-lowering agents: a nationwide cohort study of 3.6 million people. BMJ Open 2012;2: 000433 .

doi:10.1136/

bmjopen-2011-000433

- Prepublication history for this paper is available online. To view these files please visit the journal online (http:// dx.doi.org/10.1136/ bmjopen-2011-000433)

Received 5 October 2011 Accepted 10 May 2012

This final article is available for use under the terms of the Creative Commons Attribution Non-Commercial 2.0 Licence; see http://bmjopen.bmj.com

${ }^{1}$ Department of Cardiology, Gentofte Hospital, University of Copenhagen, Hellerup, Denmark

${ }^{2}$ Department of

Endocrinology,

Rigshospitalet, University of Copenhagen, Copenhagen, Denmark

${ }^{3}$ Department of Cardiology, Rigshospitalet, University of Copenhagen, Copenhagen, Denmark

${ }^{4}$ Faculty of Health Science, University of Copenhagen, Copenhagen, Denmark

Correspondence to Dr Charlotte Andersson; ca@heart.dk

\section{ABSTRACT}

Objectives: To study the association between exposures to glucose-lowering therapy and risk of cancer using the nationwide administrative registers in Denmark.

Design: Nationwide cohort study.

Setting: All hospitals in Denmark.

Participants: All individuals aged $\geq 35$ years in 1998-2009 who were naive to glucose-lowering treatment and had no history of cancer. Primary measures outcomes: first cancer diagnosis between 1998 and 2009. The RR of cancer as dependent on exposure to individual glucose-lowering agents was assessed by multivariable Poisson regression models. Results: of 159894 patients that initiated treatment with glucose-lowering agents, 12789 developed cancer, incidence rate $17.4 / 1000$ person-years. Of the remaining 3447904 individuals not using glucoselowering agents, 293878 developed cancer, incidence rate 7.9/1000 person-years. Use of different types of glucose-lowering agents including human insulin, insulin analogues, as well as sulfonylureas were associated with a quantitatively similar and significantly increased RR of cancer of 1.2-1.3 compared with unexposed individuals after multivariable adjustment. For the majority of agents, the authors identified the highest RR of cancer during the first 30 treatment days with a subsequent decline of risk approaching the cancer risk of the background population only $6-12$ months after initiation of treatments.

Conclusions: Use of most glucose-lowering agents including sulfonylureas was associated with a comparable increased risk of cancer shortly after initiation of treatment and subsequently a decline to the risk of the background population. This suggests that the relation is not causal.

\section{INTRODUCTION}

Recent epidemiological studies have reported a positive relationship between cancer and use of insulin, which has caused major safety concerns among patients as well as clinicians. ${ }^{1}$ Insulin is known to possess

\section{ARTICLE SUMMARY}

\section{Article focus}

- Several observational studies have suggested that insulin therapy may increase the risk of cancer. If insulin therapy causes/enhances cancer development, the RR would be expected to increase with longer treatment duration.

- The present study investigated the association between treatment duration with individual glucose-lowering agents and the RR of developing cancer in 3.6 million individuals.

\section{Key messages}

- Use of human insulin, insulin analogues and sulfonylureas were associated with a significantly increased RR of cancer of 1.2-1.3 compared with unexposed individuals.

- For all agents, the highest RR of cancer was found during the first 30 days of treatment. Hereafter, the RR declined rapidly, reaching a RR of cancer comparable to unexposed individuals after only 6-12 months of therapy for most agents.

- This strongly suggests that a previously reported association between use of glucose-lowering agents and increased risk of cancer is not causal.

Strengths and limitations of this study

- This study was based on complete and nationwide administrative registers, thereby reducing selection bias.

- Data on measurements on body mass index, glucose regulation and family cancer history were lacking.

affinity for the insulin-like growth factor (IGF)-1 receptor, which enhances cell growth and increases resistance to apoptosis. ${ }^{2}{ }^{3}$ Receptor binding studies demonstrated that different types of insulin analogues exhibit different affinities for the IGF-1 receptor compared with native human insulin, and studies have reported colorectal, breast and prostate cell lines to proliferate in response to exposure to some types of insulin analogues, 
but not to human insulin. ${ }^{4-6}$ To this end, some observational studies indicated that use of insulin glargine, being the approved insulin analogue with the putative highest affinity for the IGF-1 receptor, is associated with a disproportionately increased $\mathrm{RR}$ of cancer compared with human insulin. ${ }^{17-9}$

Besides insulin, other features associated with diabetes such as obesity and hyperglycaemia per se are known risk factors of cancer with increased oxidative stress and DNA damage representing suspected causal mechanisms. ${ }^{10}$ Indeed, caution is warranted concluding from observational and non-randomised studies that treatment with insulin or its analogues increases risk of cancer. Recent data have demonstrated that the RR of cancer fluctuates over time, being highest at time of diagnosis when treatment is initiated, supporting the notion of a potential observational bias. ${ }^{11}$ While much attention has been on the potential impact of insulin and insulin analogues on the risk of cancer, little is known about the time relationship between initiation of glucose-lowering treatment and cancer diagnosis for individual glucoselowering agents including oral glucose-lowering agents. We therefore examined the association between risk of incident cancer and exposure to the most used individual insulin agents (insulin glargine, insulin lispro, insulin aspart, insulin detemir and human insulin), as well as oral glucose-lowering agents (sulfonylureas (SU), metformin and thiazolidinediones) using the entire Danish population.

\section{METHODS}

Registers

The public financed healthcare system provides a unique possibility to hold complete and nationwide registers on a variety of variables in Denmark. We used four of these registers to obtain data for the present study. The Central Population Register includes information on all individuals living in Denmark. All individuals are registered with date of birth and gender. The Danish National Patient Register includes information on dates and causes of hospitalisations in Denmark since 1978. All hospitalisations are registered according to the International Classification of Diseases (ICD) system. The Danish National Prescription Register holds data on every dispensed prescription in Denmark since 1995. Dispensed drugs are registered by the Anatomical Therapeutic Classification (ATC) system with information on date of dispensing and amount dispensed available. Because of partial reimbursement of drug expenses, the accuracy of the register is very good. ${ }^{12}$ In the National Causes of Deaths Register, all deaths occurring in Denmark are registered within 2 weeks after occurrence.

\section{Study population}

We identified all Danish individuals who had not claimed a prescription for glucose-lowering agents in 1995-1997 and who had never had cancer, as deemed by any cancer diagnoses in 1978-1997, and who were $\geq 35$ years old during follow-up. Exposure to the following glucose-lowering agents was identified: insulin glargine (Lantus ${ }^{\circledR}$ ATC-code A10AE04), insulin lispro (Humalog ${ }^{\circledR}$ ATC-code A10AB04, Humalog Mix ${ }^{\circledR}$ A10AD04), human insulin (Actrapid ${ }^{\circledR}$ ATC-code A10AB01, Insulatard ${ }^{\circledR}$ ATC-code A10AC01, Mixtard ${ }^{\circledR}$ ATC-code A10AD01), insulin aspart (NovoRapid ${ }^{\circledR}$ ATCcode A10AB05, NovoMix ${ }^{\circledR}$ ATC-code A10AD05), insulin detemir (Levemir®; ATC-code A10AE05), metformin (ATC-code A10BA02), SU (ATC-code A10BB01, A10BB03, A10BB07, A10BB09, A10BB12) and thiazolidinediones (ATC-code A10BG02, A10BG03, A10BX02). Duration of treatment was calculated from dates of claimed prescriptions and the dispensed quantity of insulin agents (international units (IU)) and number and strength of tablets for oral glucose-lowering agents, respectively. Individual doses (and thus duration of treatment) were calculated by consideration of up to seven consecutive prescriptions and a continuous treatment period was assumed if this was compatible with at least the minimal daily dose ( $1 \mathrm{IU}$ for insulin agents and 0.5 tablet of oral glucose-lowering agent), as done previously. ${ }^{13-15}$ For the present study, individuals were classified as exposed between the first claimed prescription and until estimated duration of last claimed prescription.

\section{Comorbidity}

Charlson comorbidity index was used for adjustment of comorbidity in all analyses. ${ }^{16}$ The index was calculated after time had been split (see Methods section for description) using the left end point as reference. All diagnoses within 10 years prior to the left end point were considered (diabetes and cancer-related diagnoses not included).

\section{Outcomes}

The end point was any incident cancer reported in the Danish National Hospitalisation Register between 1998 and 2009 (ICD-10 codes C01-C99). Individuals were censored at time of first cancer, death or at the end of 2009. To explore whether distribution of cancers differed according to exposure to individual insulin agents, the following selected groups of cancers (according to ICD-10 codes) were identified: gastrointestinal cancers (C15-C25), lung cancer (C34), prostate cancer (C61), breast cancer (C50), gynaecological cancers (C51-C57) and urological cancers (C64-C67). It should be noted that Denmark possesses a specific national cancer register, in which additional information on, for example, tumour stages is available. The cancer register is mainly based on the national patient register, but some of the diagnoses are manually reviewed in the cancer register, resulting in a discard of a minor part of the cancer diagnoses from the national patient register. For the present analyses, the national patient register was nevertheless considered sufficient in order to investigate the aims of the present paper. We did compare our 
end points with the diagnoses in the cancer register (we had data available for 1998-2006 from this latter register) and found a concordance rate exceeding $90 \%$.

\section{Statistics}

The RR of cancer as dependent on use and duration of individual glucose-lowering pharmacotherapy was examined with multivariable Poisson regression models. The whole cohort was included on 1 January 1998 or at their 35th birthday if younger than 35 years at 1 January 1998.

The lexis-macro (http://192.38.117.59/ bxc/Lexis/ Lexis.sas; last accessed 8 January 2012) was used for all analyses and included two time scales: calendar time (bands were split in 1-year periods after 1 January 1998) and duration of glucose-lowering treatment (bands were split at every change in glucose-lowering treatment regimens as well at $1,3,6,12$ months and every year hereafter). Dichotomous variables were hereafter created for the use of glucose-lowering agents (current use or no use; left end point as reference). Similarly, dichotomous variables were created for use of glucoselowering agents in specific time intervals (eg, 'use of actrapid day $0-30$ ' yes vs no; left end point as reference). Age and comorbidity scores were calculated at the beginning of each interval. For adjustment for the effects of age in Poisson analyses, age was round in 0.5year intervals.

Two different multivariable Poisson analyses (both being adjusted for Charlson comorbidity score, gender, age and calendar year) were performed. In the first analysis, RRs associated with current use of the different glucose-lowering agents were explored. In the second analysis, the importance of time since initiation of the different agents was explored (variables were included as use vs no use of a specific agent in a specific time window (eg, 'actrapid day 0-30', 'humalog day 365.25-730.5' etc)).

Calculations were performed using SAS V.9.2 (SAS institute).

\section{RESULTS}

Of totally 3607798 individuals included, 159894 initiated treatment with glucose-lowering agents. Table 1 presents total numbers of individuals ever exposed and the cumulative exposure time for individual glucoselowering agents. The most frequently used agents included human insulin and oral glucose-lowering agents. Insulin lispro had a low numbers of users and contributed only with a low cumulative exposure time.

\section{RR of cancer}

Crude incidence rates, numbers and cancer types are shown in table 1 . The distributions of cancer types were similar within the different exposure groups and in the unexposed population group. In multivariable analysis, male gender, increasing age (up to 85 years) and increasing Charlson comorbidity scores were found to be associated with an increased risk of cancer. Figure 1 presents the rate ratio (RR) of cancer associated with use of individual glucose-lowering agents. Besides metformin, most agents were associated with a significant increase in RR compared with the background population. Pooling all insulin analogues into one group and all human insulin agents into another group gave similar results: RR of cancer for use of human insulin was 1.40 (95\% CI 1.33 to 1.48 ) and for use of insulin analogues, it was 1.14 (1.05 to 1.23). As illustrated in figure $2 \mathrm{~A}-\mathrm{C}$, there was a significant fluctuation in $\mathrm{RR}$ of cancer over time. The first 30-day period after initiation of glucose-lowering treatment was associated with a very pronounced increase in RR of cancer for most agents, which subsequently declined rapidly during the first year of treatment, resulting in a RR of 1 after approximately a half to 1 year of treatment.

\section{DISCUSSION}

In the present nationwide study including more than 3.5 million unselected individuals aged $\geq 35$ years who were naive to glucose-lowering pharmacotherapy at study baseline, we found that use of several different types of glucose-lowering agents, including use of the oral glucose-lowering agent group SU, were associated with an increase in RR of all types of cancer of approximately $20 \%-30 \%$. Importantly, this risk was found to be highly fluctuating over time with, for example, more than a twofold increase in RR of cancer already during the first 30 days after treatment initiation with insulin. Interestingly, the RR of being diagnosed with cancer declined significantly within the first year after onset of treatment down to that of the background population for all the studied agents. Data from the present nationwide study therefore suggest that the previously reported associations between an increase in risk of cancer and use of insulin are most likely to be driven by confounding and/or surveillance bias.

From the present study, it was impossible to investigate what may have caused the findings, but it is known that hyperglycaemia and cancer are more commonly seen together than expected by chance. ${ }^{7}$ In our study population, initiation and use of glucose-lowering therapy may have mirrored poor glycaemic control, occult cancer or both. Hyperglycaemia has previously been shown to increase the risk of several cancer types. ${ }^{17} 18$ Elevated stress levels, as seen in many cancer types, are associated with increasingly fasting serum glucose levels ${ }^{19}$ and patients with cancer are often insulin resistant, ${ }^{20}$ which may unmask overt diabetes in predisposed individuals. The immediate risk of cancer on start of insulin therapy is therefore likely to be driven by diagnostic investigation when one of the diagnoses was made. A true risk of cancer caused by insulin therapy would be expected to remain high or even increase over time.

The present study lacked data on several important variables such as body mass index, HbA1c concentrations, 


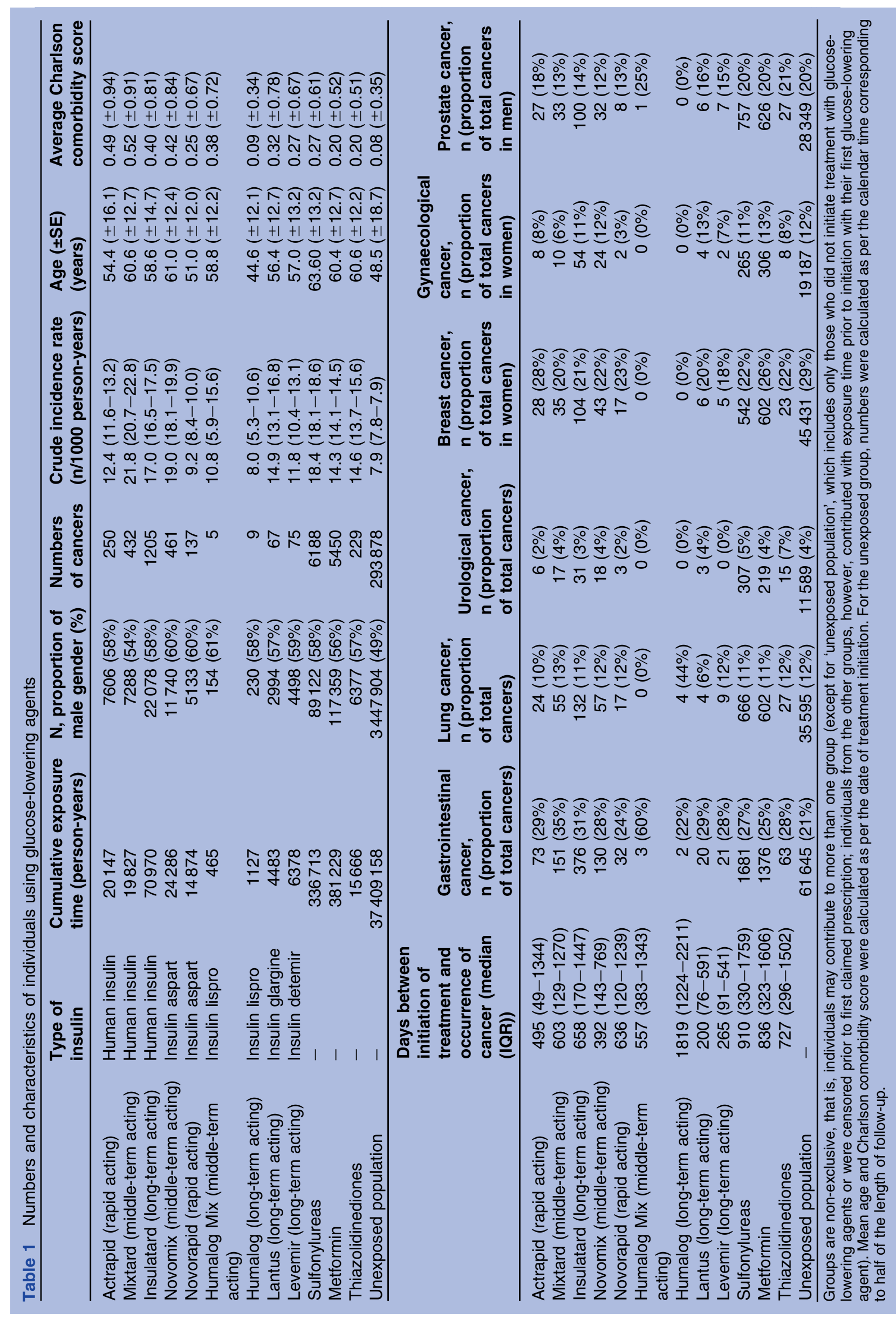




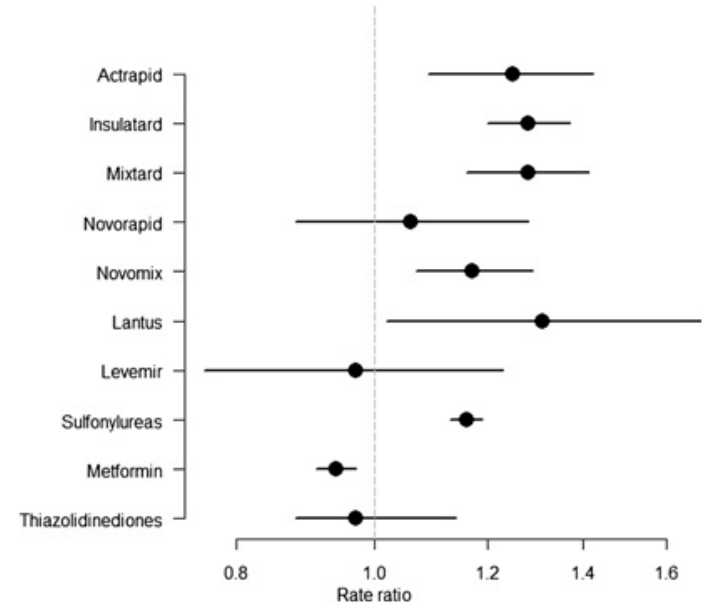

Figure 1 RR of cancer according to exposure to individual glucose-lowering agents.

physical activity, family cancer history, alcohol consumption, dietary pattern and smoking habits, and it was not designed or powered to answer questions about the risk of different cancer subtypes or about the safety profiles of individual insulin analogues. As for the risk of specific cancers with glucose-lowering treatments, it must be emphasised that the pattern of cancer subtypes was similar within all treatment subgroups and the control group who were not on any glucose-lowering therapy. Thus, even though we cannot exclude having overlooked associations between treatments and risk of any cancer subtypes, it seems unlikely that any quantitative major causal associations have been overlooked. Of particular relevance, the previously raised concerns about the risk associated with use of insulin glargine could not be thoroughly investigated in the present study because we had rather few individuals using this agent and very few patients using glargine developed cancers, as compared with previous studies. ${ }^{1} \quad 8 \quad 9$ Furthermore, patients were only considered at risk, while using a specific agents meaning that cancers occurring after treatment cessation with a specific agent may be overlooked. Nevertheless, it is interesting to speculate whether and the extent to which the time from initiation of glucose-lowering treatment may have biased the findings of previous studies. At least when considering the time of the observation period, insulin glargine was-and to some extent still is-a relatively new agent on the market, and it is likely that patients with newonset diabetes were more prone to initiate treatment with insulin glargine compared with patients being well regulated on older agents. In this context, a Swedish study did not exclude patients with prior use of insulin, and during a short follow-up time of maximally 2 years, they found that use of insulin glargine as monotherapy was associated with a twofold increase in the risk of breast cancer as compared with use of other types of insulin. ${ }^{9}$ Similar, a German study had a significantly longer follow-up time for patients on human insulin than for patients on insulin glargine, which may have contributed to their findings of an increased risk of cancer associated with insulin glargine. ${ }^{8}$ In a third Scottish study investigating the risk of cancer as dependent on insulin glargine, on the other hand, three specific exposure groups were identified: insulin glargine monotherapy, insulin glargine + non-glargine insulin and non-glargine insulin monotherapy. ${ }^{1}$ Compared with the other two groups, the insulin glargine monotherapy group had significantly shorter treatment duration prior to study start. The study demonstrated no excess increase in risk of cancer for the use of insulin glargine, but when restricting the analysis to patients on insulin glargine as monotherapy, these patients were found to have a higher RR of all cancers as
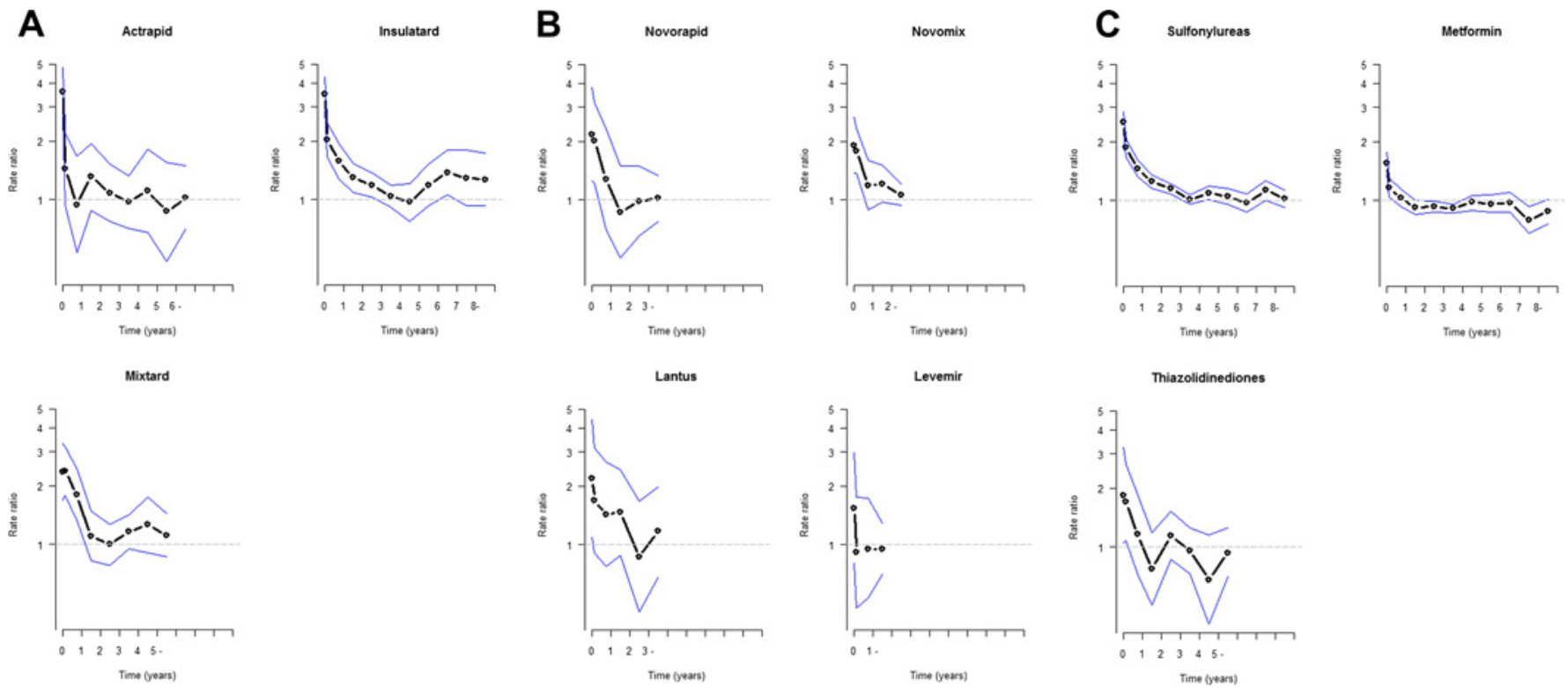

Figure 2 RR of cancer according to exposure length of individual glucose-lowering agents: (A) human insulin; (B) insulin analogues; (C) oral agents. 
well as breast cancers compared with patients on nonglargine insulin. The authors concluded that their finding may be due to allocation bias rather than an effect of insulin glargine itself, which our data support. Altogether, more studies are needed to answer this question, and in particular, our results demonstrate that it is of crucial importance to take treatment duration into account, in order to eliminate confounding-byindication/surveillance bias.

As also shown in at least one previous study, ${ }^{21}$ another interesting finding of this study was that use of SU was associated with a risk of cancer quantitatively similar to that of insulin in overall analysis. While it theoretically could be speculated that the effect of SU's to increase insulin levels in plasma could represent a causal mechanism, the finding that this excess risk diminished with duration of treatment, mirroring the relationship between insulin and cancer, makes this highly unlikely to be the case. Indeed, this finding is fully in agreement with the idea that newly diagnosed diabetes patient are more often to be diagnosed as having cancer, and vice versa, all together supporting that the association between glucose-lowering agents and cancer at least to some significant extent may represent confounding. Finally, very recent data from the Food and Drug Administration side effect registry reported increased risk of some types of cancer in patients treated for relatively short periods with the novel glucagon-like peptide (GLP)-based therapies. ${ }^{22}$ The current data raise the possibility that confounding and lack of correction for time of treatments to some unknown extent may explain the risk of cancers associated with GLP-1-based therapies.

\section{CONCLUSIONS}

Use of most glucose-lowering agents including insulin was associated with an overall increased risk of cancer. However, this increase was due to an association observed early in treatment only, suggesting that the association may not be causal. More studies are warranted to further investigate this hypothesis.

Acknowledgements The authors would like to thank Thomas Alexander Gerds, Dr rer. nat, Department of Public Health, Section of biostatistics, Copenhagen University, for statistical advises throughout the revision process of the paper.

Contributors All authors contributed to conception and design. C.A. and C T.-P. performed the data analyses. C.A. and C.T.-P. had full access to all of the data in the study and take responsibility for the integrity of the data and the accuracy of the data analysis. C.A., A.V. and C.T.-P. drafted the article and all authors revised it critically for important intellectual content (C.A., A.V., C.S., M.S., R.S., J.L., G.H.G., L.K. and C.T.-P.). All authors approved the final version to be published.

Funding This research received no specific grant from any funding agency in the public, commercial or not-for-profit sectors.
Competing interests All authors report no potential conflicts of interest, but it should be mentioned that Dr AV was previously employed by Steno Diabetes Center, which is owned by Novo Nordisk.

Provenance and peer review Not commissioned; externally peer reviewed.

Data sharing statement Unfortunately not doable.

\section{REFERENCES}

1. Colhoun HM; SDRN Epidemiology Group. Use of insulin glargine and cancer incidence in Scotland: a study from the Scottish diabetes research Network Epidemiology group. Diabetologia 2009;52:1755-65

2. Macaulay VM. Insulin-like growth factors and cancer. $\mathrm{Br} \mathrm{J}$ Cancer 1992:65:311-20.

3. Samani AA, Yakar S, LeRoith D, et al. The role of the IGF system in cancer growth and metastasis: overview and recent insights. Endocr Rev 2007;28:20-47.

4. Kurtzhals $P$, Schaffer L, Sorensen A, et al. Correlations of receptor binding and metabolic and mitogenic potencies of insulin analogs designed for clinical use. Diabetes 2000;49:999-1005.

5. Smith U, Gale EA. Does diabetes therapy influence the risk of cancer? Diabetologia 2009;52:1699-708.

6. Weinstein D, Simon M, Yehezkel E, et al. Insulin analogues display IGF-I-like mitogenic and anti-apoptotic activities in cultured cancer cells. Diabetes Metab Res Rev 2009;25:41-9.

7. Giovannucci E, Harlan DM, Archer MC, et al. Diabetes and cancer: a consensus report. CA Cancer J Clin 2010;60:207-21.

8. Hemkens LG, Grouven U, Bender R, et al. Risk of malignancies in patients with diabetes treated with human insulin or insulin analogues: a cohort study. Diabetologia 2009;52:1732-44.

9. Jonasson JM, Ljung R, Talback M, et al. Insulin glargine use and short-term incidence of malignancies-a population-based follow-up study in Sweden. Diabetologia 2009;52:1745-54.

10. Maritim AC, Sanders RA, Watkins JB 3rd. Diabetes, oxidative stress, and antioxidants: a review. J Biochem Mol Toxicol 2003; 17:24-38.

11. Carstensen B, Witte DR, Friis S. Cancer occurrence in Danish diabetic patients: duration and insulin effects. Diabetologia 2012;55:948-58.

12. Gaist D, Sorensen HT, Hallas J. The Danish prescription registries. Dan Med Bull 1997;44:445-8.

13. Andersson C, Olesen JB, Hansen PR, et al. Metformin treatment is associated with a low risk of mortality in diabetic patients with heart failure: a retrospective nationwide cohort study. Diabetologia 2010;53:2546-53.

14. Sorensen R, Hansen ML, Abildstrom SZ, et al. Risk of bleeding in patients with acute myocardial infarction treated with different combinations of aspirin, clopidogrel, and vitamin $\mathrm{K}$ antagonists in Denmark: a retrospective analysis of nationwide registry data. Lancet 2009;374:1967-74.

15. Fosbol EL, Gislason GH, Jacobsen S, et al. Risk of myocardial infarction and death associated with the use of nonsteroidal antiinflammatory drugs (NSAIDs) among healthy individuals: a nationwide cohort study. Clin Pharmacol Ther 2009;85:190-7.

16. Charlson ME, Pompei $\mathrm{P}$, Ales $\mathrm{KL}$, et al. A new method of classifying prognostic comorbidity in longitudinal studies: development and validation. J Chronic Dis 1987;40:373-83.

17. Jee $\mathrm{SH}$, Ohrr $\mathrm{H}$, Sull JW, et al. Fasting serum glucose level and cancer risk in Korean Men and Women. JAMA 2005;293:194-202.

18. Stattin $\mathrm{P}, \mathrm{Björ} \mathrm{O}$, Ferrari $\mathrm{P}$, et al. Prospective study of hyperglycemia and cancer risk. Diabetes care 2007;30:561-7.

19. Halter JB, Beard JC, Porte D Jr. Islet function and stress hyperglycemia: plasma glucose and epinephrine interaction. $A m \mathrm{~J}$ Physiol 1984;247:E47-52.

20. Poulson J. The management of diabetes in patients with advanced cancer. J Pain Symptom Manage 1997;13:339-46.

21. Currie CJ, Poole CD, Gale EA. The influence of glucose-lowering therapies on cancer risk in type 2 diabetes. Diabetologia 2009;52:1766-77.

22. Elashoff M, Matveyenko AV, Gier B, et al. Pancreatitis, pancreatic, and thyroid cancer with glucagon-like peptide-1-based therapies. Gastroenterology 2011;141:150-6. 